\title{
A Randomized Controlled Trial of IVR-Based Alcohol Brief Intervention to Promote Patient-Provider Communication in Primary Care
}

\author{
Gail L. Rose, Ph.D. ', Gary J. Badger, M.S. ${ }^{2}$, Joan M. Skelly, M.S. ${ }^{2}$, Tonya A. Ferraro, M.Ed. ${ }^{3}$, \\ Charles D. MacLean, M.C.M.D. ${ }^{4}$, and John E. Helzer, M.D. ${ }^{7}$
}

'Department of Psychiatry, The University of Vermont, Burlington, VT, USA; ${ }^{2}$ Department of Medical Biostatistics, The University of Vermont, Burlington, VT, USA; ${ }^{3}$ Department of Research Administrative Services, Harvard University, Cambridge, MA, USA; ${ }^{4}$ Department of Medicine, The University of Vermont, Burlington, VT, USA.

\begin{abstract}
BACKGROUND: Brief interventions for unhealthy drinking in primary care settings are efficacious, but underutilized. Efforts to improve rates of brief intervention though provider education and office systems redesign have had limited impact. Our novel brief intervention uses interactive voice response (IVR) to provide information and advice directly to unhealthy drinkers before a physician office visit, with the goals of stimulating in-office dialogue about drinking and decreasing unhealthy drinking. This automated approach is potentially scalable for wide application.
\end{abstract}

OBJECTIVE: We aimed to examine the effect of a pre-visit IVR-delivered brief alcohol intervention (IVR-BI) on patient-provider discussions of alcohol during the visit.

DESIGN: This was a parallel group randomized controlled trial with two treatment arms: 1) IVR-BI or 2) usual care (no IVR-BI).

PARTICIPANTS: In all, 1,567 patients were recruited from eight university medical center-affiliated internal medicine and family medicine clinics.

INTERVENTIONS: IVR-BI is a brief alcohol intervention delivered by automated telephone. It has four components, based on the intervention steps outlined in the National Institute of Alcohol Abuse and Alcoholism guidelines for clinicians: 1) ask about alcohol use, 2) assess for alcohol use disorders, 3) advise patient to cut down or quit drinking, and 4) follow up at subsequent visits.

MAIN MEASURES: Outcomes were patient reported: patient-provider discussion of alcohol during the visit; patient initiation of the discussion; and provider's recommendation about the patient's alcohol use.

KEY RESULTS: Patients randomized to IVR-BI were more likely to have reported discussing alcohol with their provider (52\% vs. $44 \%, p=0.003$ ), bringing up the topic themselves (20\% vs. $12 \%, p<0.001)$, and receiving a recommendation ( $20 \%$ vs. $14 \%, p<0.001)$. Other predictors of outcome included baseline consumption, education, age, and alcohol use disorder diagnosis.

CONCLUSIONS: Providing automated brief interventions to patients prior to a primary care visit promotes discussion about unhealthy drinking and increases specific professional advice regarding changing drinking behavior.

Received September 30, 2015

Revised February 23, 2016

Accepted March 23, 2016

Published online May 20, 2016
KEY WORDS: physician-patient relations; alcohol consumption; brief intervention; primary care; interactive voice response; automated intervention; automated telephone; patient engagement. J Gen Intern Med 31(9):996-1003

DOI: $10.1007 / \mathrm{s} 11606-016-3692-4$

(c) Society of General Internal Medicine 2016

\section{INTRODUCTION}

Brief alcohol interventions (BIs) in primary care settings for patients with unhealthy drinking are underutilized for many reasons including time constraints, workflow incompatibility, lack of ancillary resources, and stigmatization. ${ }^{1-4}$ Efforts to increase the delivery of BIs through an exclusive focus on provider training or incentives has had limited impact on intervention rates. ${ }^{5-7}$ In two of our previous studies, approximately half of patients interviewed after a primary care visit reported that they themselves had brought up the topic of alcohol for discussion. ${ }^{8,9}$

With the dual objective of increasing patient-provider communication about alcohol and offering an automated pre-visit $\mathrm{BI}$ to patients who might not otherwise receive one, we developed a system for alcohol screening and BI using Interactive Voice Response (IVR). The IVR performs pre-visit screening to identify individuals with unhealthy alcohol consumption, ${ }^{10}$ offers a BI to those who screen positive, and encourages them to discuss alcohol at the upcoming visit. The current report focuses on evaluating the IVR-BI as an instrument for stimulating in-office dialogue about drinking behavior.

Small-scale pilot studies previously showed the feasibility of our IVR Screen ${ }^{11}$ and BI. ${ }^{9}$ Consequently, we conducted a randomized, controlled trial in a large primary care network to evaluate the effects of IVR-BI on patient-provider communication (Aim 1) and on subsequent alcohol consumption (Aim $2)$. The current report focuses on Aim 1; clinical results corresponding to Aim 2 are forthcoming. Aim 1 hypotheses were that individuals randomized to complete the IVR-BI would be more likely on the upcoming visit to 1) discuss drinking with their provider, 2) self-initiate that discussion, and 3) receive an alcohol-related recommendation from their provider. We further examined the specificity of the IVR-BI to 
stimulate discussions of alcohol, compared to other behavioral health topics included in the screen, but not targeted by the IVR-BI.

\section{METHODS}

\section{Sample and Setting}

Eligibility criteria were as follows: scheduled for a routine (non-acute) primary care visit in the next 3 days, spoke English, had no cognitive or hearing deficits, and screened positive for unhealthy alcohol use.

The recruitment sites were urban $(n=1)$, suburban $(n=5)$ and rural $(n=2)$ primary care outpatient clinics affiliated with the academic medical center, with four to 12 providers each.

\section{Design}

This parallel group randomized controlled trial had two arms, IVR-BI and control (no IVR-BI). After screening, all participants were randomized by the IVR system based on a random number generator to either complete or not complete the IVRBI. Participants in the IVR-BI group were seamlessly connected to the IVR-BI after randomization. A post-visit telephone interview (PVI) was conducted a few days after the clinic visit. Because this interview included questions about the IVR system, research assistants (RAs) conducting the interviews were not blind to study condition. All participants received $\$ 10$ for completing the IVR randomization call and $\$ 20$ for the PVI, regardless of randomization condition.

Primary care providers (PCPs) were blind to patients' participation in the trial. Our research question was whether a patient-directed intervention could affect the care patients received, not whether providers could be externally influenced to make changes in their care. PCPs were free to provide their "usual care," which may or may not include alcohol screening, discussion, BI, or referral.

\section{Recruitment Procedures}

Screening was done by phone two to three days before participants' medical appointments. Patients completed an IVR Screen that included the Single Alcohol Screening Question (SASQ); "How many times in the past year have you had five (for men; four for women) drinks in one day?", ${ }^{12}$ along with single items assessing pain, smoking, exercise, weight, and mood. A previous publication describes the IVR Screen in detail. ${ }^{10}$ Respondents had the option of routing their IVR Screen results immediately to their Electronic Medical Record (EMR), which $95 \%$ elected to do. Results were viewable by PCPs in the EMR and consisted of a simple list of each of the six items and the patient's response.

Respondents answering one or more on the $\mathrm{SASQ}^{12}$ were eligible to enroll in the trial. An IVR intervention was only available for alcohol, not for the other IVR Screen topics. Upon completion of the IVR Screen, eligible respondents were transferred to a RA for verbal consent and study invitation. Consenting patients were scheduled for the PVI to occur as soon after the medical visit as schedules allowed, then were transferred to the IVR-BI. Participants could schedule the IVR-BI for another time, so long as it was prior to the office visit. After transferring a patient, the RA could monitor their connection to the IVR line. If a disconnect occurred, the RA would call the patient immediately and offer to re-connect them. All procedures were approved by the University of Vermont Human Subjects Committee and the Medical Center's Risk Management Department.

\section{IVR-BI Intervention}

The IVR-BI begins with brief instructions on keypad entries, a reminder that it is part of a research project, and assurance of privacy and confidentiality. The IVR-BI content is based on National Institute on Alcohol Abuse and Alcoholism (NIAAA) ${ }^{13}$ recommendations (see Fig. 1). The "Ask" element is accomplished with the SASQ ${ }^{12}$ from the IVR Screen. The IVR-BI itself begins by defining standard drink equivalences, i.e., that one beer, one glass of wine, and one shot or mixed drink have about the same amount of alcohol. Two quantity /frequency questions follow, then NIAAA safe-drinking guidelines are stated (maximum 14 drinks/week, fewer than five drinks/occasion for men younger than 65 ; seven/week, fewer than four/occasion for women or individuals aged 65 or older).

The "Assess" section screens for Alcohol Use Disorder (AUD) because a full diagnostic assessment of AUD by IVR was not feasible. The screening consists of two DSM-IV criteria shown to correlate highly with diagnosis, ${ }^{14}$ i.e., use in hazardous situations and drinking larger amounts or for a longer period of time than intended. This section also includes a question about prior withdrawal experiences. Positive responses to any AUD screening or withdrawal question is followed by advice to seek an evaluation by a doctor or alcohol specialist, and a statement that doctors typically recommend quitting drinking for people with these symptoms. The screen-positive advice cautions patients to discuss any quit attempt with a doctor to avoid dangerous withdrawal.

The "Advise and Assist" section begins with a readiness to change assessment, then branches accordingly. The NotReady branch offers three "Readiness Suggestions" then plays a final reminder about the research protocol. The Ready branch leads to a choice to hear guidance on cutting down and/or quitting. Advice for Cutting Down includes goal-setting, planning for urges and high-risk situations, proactive avoidance of triggers, self-monitoring, and other strategies. The Advice to Abstain section describes treatment and mutual-help models commonly used to achieve abstinence, and includes information on local support and treatment resources.

For all callers, the IVR-BI ends with a section on "Followup Support": Callers are encouraged to talk with their PCP 


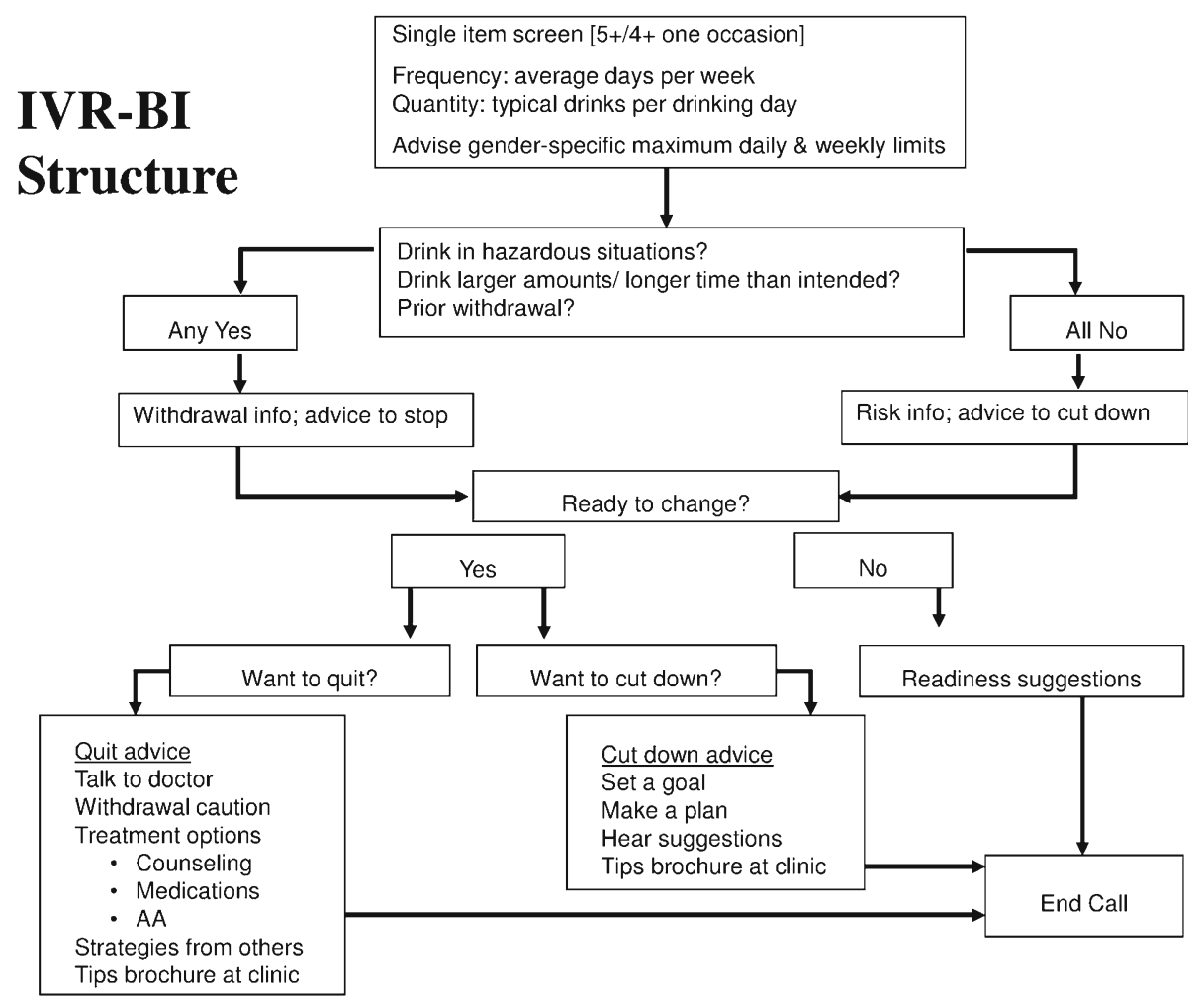

Figure 1. Branching logic used to program the IVR-BI

about their alcohol use and to avail themselves of patient education materials at their doctor's office. The full text of the IVR-BI and IVR Screen is available from the authors.

\section{Assessments}

PVIs assessed in-visit doctor-patient communication, baseline demographics, AUD diagnosis, and recent history of alcohol use. Interviews took approximately 30 minutes.

Outcome Measures. Doctor-patient communication about alcohol use was assessed with three questions: 1) "Did you talk with the doctor about alcohol use?" 2) "Who brought it up?" and, 3) "Did [provider name] make any recommendations about your alcohol use?" This brief assessment is similar to other one- to two-item patient surveys that have been effectively used to document alcohol BI occurrence in other primary care research, ${ }^{15,16}$ and that have been used in the VA Health Care system as a performance monitoring method. ${ }^{17}$

To determine if the IVR-BI effect on patient-provider discussion would be specific to alcohol, we assessed patientprovider discussions of other topics covered in the six-item IVR Screen that patients had completed during recruitment for the randomized trial, i.e., pain, smoking, drinking, exercise, weight, and mood. The same three questions about discussion, advice, and recommendation were asked about each of the screening items.

Baseline Measures. Baseline alcohol consumption during the time period of 30 days prior to the primary care visit up through the day preceding the PVI was ascertained using The Timeline Followback (TLFB) $)^{18}$ calendar method.

Past-year DSM-IV AUD was assessed using the Alcohol Use Disorders section of the Composite International Diagnostic Interview - Substance Abuse Module (CIDI-SAM), ${ }^{19} \mathrm{a}$ structured interview designed for administration by trained lay interviewers for the assessment of substance use disorders according to DSM-IV diagnostic criteria.

Participants randomized to the IVR-BI condition were interviewed about their subjective experience using the IVR-BI. Participants were asked about the effect of the IVR-BI on their awareness of drinking or interest to change, and how the IVRBI compared to any information or advice they had received from the PCP.

\section{Statistical Methods}

Baseline comparisons between treatment groups on demographic and alcohol use variables were performed using chi square, t-tests, and Wilcoxon Rank Sum tests. MantelHaenszel chi square tests were used to compare treatment groups on the primary outcome measures, stratifying on whether patients met criteria for an AUD. Breslow-Day tests were used to test for heterogeneity of the treatment effect between patients who did and did not meet criteria for an AUD. Primary outcome measures were: 1) percent of patients who reported a discussion about alcohol with their provider, 2) percent who reported self-initiating a discussion about alcohol with their provider, and 3) percent who reported receiving a 
Table 1. Characteristics of Participants in IVR-BI Randomized Trial

\begin{tabular}{|c|c|c|c|}
\hline & Control $(N=783)$ & IVR-BI $(N=784)$ & $p$ value \\
\hline$\%$ Female & 53 & 51 & 0.46 \\
\hline \multicolumn{4}{|l|}{ Marital Status } \\
\hline$\%$ single & 30 & 34 & \multirow[t]{4}{*}{0.17} \\
\hline$\%$ married/civil union & 57 & 55 & \\
\hline$\%$ divorced/separated & 11 & 8 & \\
\hline$\%$ widowed & 2 & 3 & \\
\hline$\%$ White & 95 & 96 & 0.39 \\
\hline \multicolumn{4}{|l|}{ Age } \\
\hline$\% 18-29$ & 18 & 19 & \multirow[t]{4}{*}{0.81} \\
\hline$\% 20-44$ & 25 & 25 & \\
\hline$\% 45-64$ & 40 & 40 & \\
\hline$\% 65+$ & 17 & 16 & \\
\hline \multicolumn{4}{|l|}{ Education } \\
\hline$\%<$ High School Diploma or GED & 30 & 30 & \multirow[t]{3}{*}{0.84} \\
\hline$\%$ Some College /Associates degree & 10 & 11 & \\
\hline$\%$ Bachelor's Degree or greater & 60 & 59 & \\
\hline Number of times drank $5+/ 4+$ on one occasion in past year, median (IQR) & $5(2-10)$ & $4(2-11)$ & $0.37 *$ \\
\hline$\%$ meeting criteria for Alcohol Use Disorder & 28 & 28 & 0.79 \\
\hline$\%$ reported drinking $5+/ 4+$ in the past 30 days & 38 & 36 & 0.39 \\
\hline Number of drinks per week & $7.9 \pm 10.1$ & $7.4 \pm 8.9$ & 0.34 \\
\hline Number of drinking days per week & $3.0 \pm 2.4$ & $2.9 \pm 2.3$ & 0.67 \\
\hline Number of drinks per drinking day & $2.6 \pm 2.0$ & $2.5 \pm 1.7$ & 0.25 \\
\hline
\end{tabular}

Note: Values represent mean $\pm S D$, unless otherwise specified. Statistical significance is based on t-tests for continuous measures and chi-square tests for categorical variables

*Significance level based on Wilcoxon Rank Sum test

recommendation about alcohol from their provider. Stepwise logistic regression was used to construct multivariate models predicting each outcome, with baseline characteristics (see Table 1) and treatment condition as potential predictors. For characteristics that were significant predictors, terms representing interactions with treatment condition were subsequently examined to determine whether their effects were treatment specific.
Additionally, chi square tests were used to compare treatment groups on the percent of participants available for follow-up and the percent of patients who discussed other screening items not targeted by the intervention (i.e., pain, smoking, exercise, weight, and mood). These latter comparisons were limited to subjects who endorsed the specific item at the time of their screen. All analyses were performed using SAS statistical software Version 9.4. ${ }^{20}$ The study was

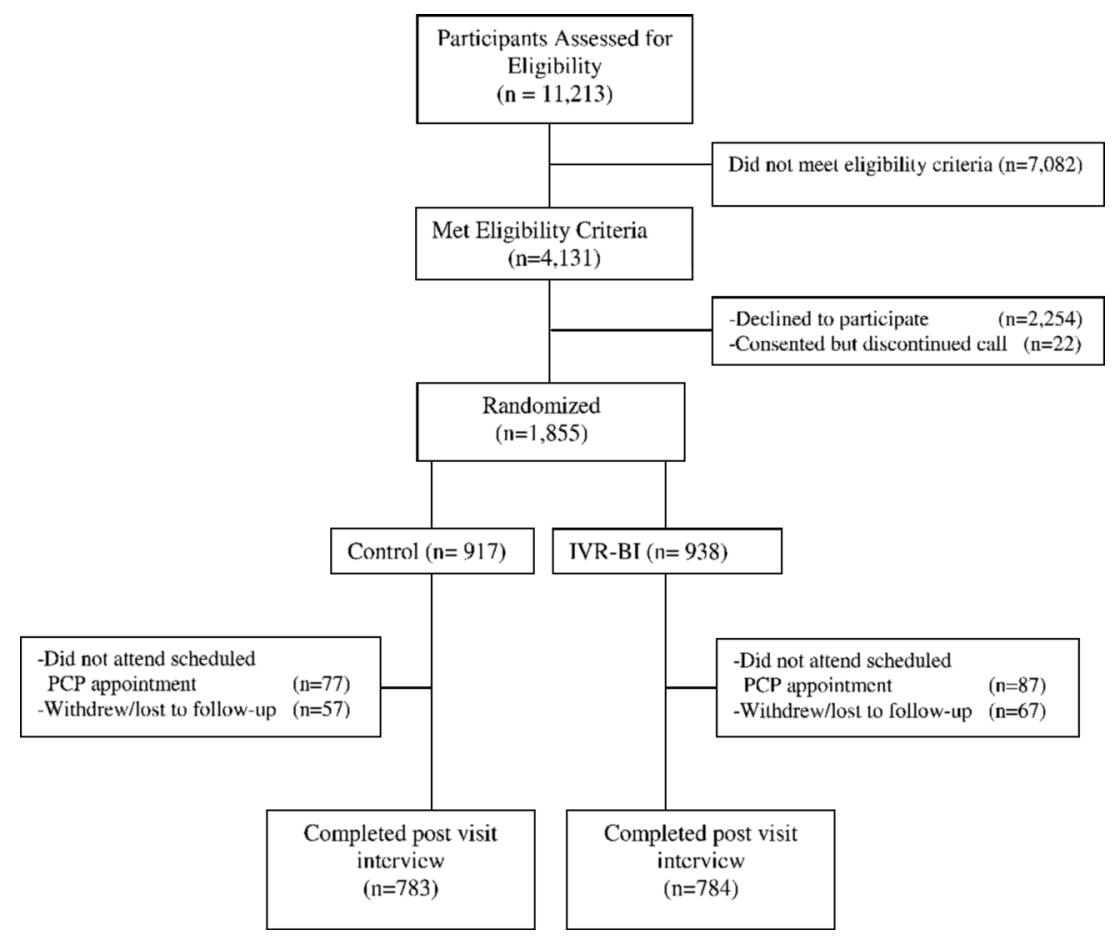

Figure 2. CONSORT diagram showing the flow of participants. 
designed to enroll approximately 700 evaluable subjects per group, which results in power greater than $95 \%$ using $\alpha=0.05$ to detect an absolute difference of $10 \%$ for each of the primary outcome measures for a wide range of underlying percentages.

\section{RESULTS}

As shown in Figure 2, 11,213 participants completed the IVRScreen from June 2012 to January 2015. Fifty-nine providers (21 male, 39 female; 49 physicians, ten mid-level providers) referred patients to the study [median 28 referrals per provider (IQR 10-46)]. A total of 1,855 adults aged 18-87 years were subsequently randomized.

The IVR-BI completion rate was $97 \%$. PVI data were available for 1,567 patients ( $84 \%$ ), with equivalent percentages in the IVR and control groups ( $84 \%$ and $85 \%$ respectively, $p=0.28)$. The PVI occurred a median of one day $(\mathrm{IQR}=0$ to 3 days) after the medical visit. Demographic characteristics and baseline alcohol use did not significantly differ between randomized groups (Table 1).

Significant differences between groups were observed on the three primary outcome measures (Fig. 3). Compared to controls, a higher percentage of participants randomized to IVR-BI reported having a discussion with their PCP about drinking [52\% of IVR-BI group vs. $44 \%$ of controls; $\mathrm{OR}=1.36,95 \% \mathrm{CI}: 1.11-1.66, p=0.003]$. Significantly more IVR-BI (20\%) than control (12\%) subjects reported self-initiating a discussion $[\mathrm{OR}=1.89,95 \% \mathrm{CI}: 1.43-2.50$, $p<0.001$ ], and receiving a recommendation [20\% IVR-BI vs. $14 \%$ control; $\mathrm{OR}=1.59,95 \%$ CI: $1.21-2.08$, $p<0.001]$. Meeting AUD criteria was associated with increased likelihood of all three outcomes [Discussion $54 \%$ vs. $46 \%, \mathrm{OR}=1.4,95 \% \mathrm{CI}: 1.12-1.75, p=0.003$; SelfInitiation $20 \%$ vs. $15 \%, \mathrm{OR}=1.50,95 \% \mathrm{CI}: 1.12-2.00$, $p=0.003$; Recommendation $29 \%$ vs. $13 \%, \mathrm{OR}=2.82$, $95 \%$ CI: $2.14-3.70, p<0.001]$. However, there was no evidence that treatment differences were dependent on AUD status [Discussion, $p=0.86$; Self-Initiation, $p=0.16$; and Recommendation, $p=0.61]$.

Multivariate results based on stepwise logistic regression are summarized in Table 2. When baseline characteristics and treatment condition were considered as candidates for predicting outcome, treatment condition remained a significant predictor of all three outcomes, with IVR-BI associated with increased odds of having a discussion, self-initiating, and receiving a recommendation. In addition, baseline consumption (i.e., drinks/week) was predictive of all three outcomes, with increased consumption associated with increased odds. Other predictors were specific to outcome, with increased education associated with increased odds of discussion, increased age associated with greater self-initiation, and AUD predictive
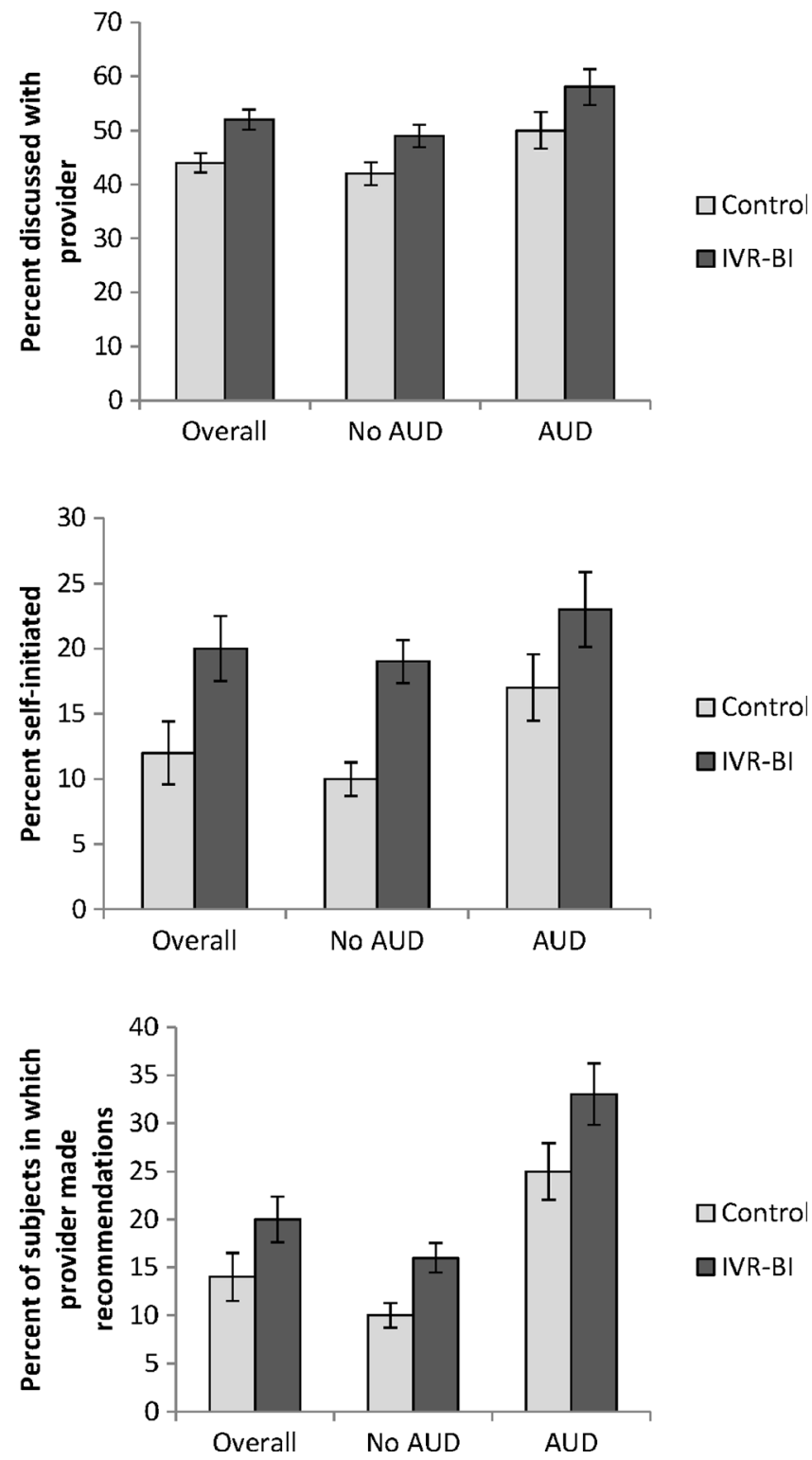

Figure 3. Group comparisons on communication outcome variables. Differences between groups were significant for discussion of alcohol, $p=0.003$; self-initiate, $p<0.001$; and physician recommendation, $p<0.001$. AUD $=$ Alcohol Use Disorder.

of receiving a recommendation. There was no evidence that the predictors were dependent on treatment condition.

Table 3 displays the percentage of participants who reported discussion of each of the IVR Screen items with their PCP. Alcohol was the only item that showed a significant group difference. Pain, tobacco use, physical activity, weight, and mood were each discussed between $72 \%$ and $91 \%$ of the time, independent of group assignment. These results highlight the specificity of the IVR-BI effect.

A majority (55\%) of IVR-BI participants said the treatment made them more aware of how much they drink, and $25 \%$ said it motivated them to make changes. Among those who also had a discussion with the provider, $37 \%$ said the IVR-BI was more useful than the provider discussion. 
Table 2. Results of Logistic Regressions Predicting Primary Outcome Measures from Baseline Characteristics and Treatment Condition

\begin{tabular}{|c|c|c|c|c|c|c|}
\hline \multicolumn{7}{|l|}{ Dependent Variable } \\
\hline & Discussion & & Self-Initiatior & & $\begin{array}{l}\text { Provider } \\
\text { Recommenc }\end{array}$ & \\
\hline \multirow[t]{2}{*}{ Explanatory Variable } & OR & & OR & & OR & \\
\hline & $(95 \% \mathrm{CI})$ & $p$ & $(95 \% \mathrm{CI})$ & $p$ & $(95 \% \mathrm{CI})$ & $p$ \\
\hline $\begin{array}{l}\text { Group } \\
\text { (IVR vs. Control) } \\
\text { Drinks per Week } \\
\text { (per 5-drink increase) } \\
\text { Education } \\
\text { ( } \geq \text { Bachelor Degree vs. }<\text { Bachelor Degree) } \\
\text { Age } \\
\text { (per 5-year increase) } \\
\text { Alcohol Use Disorder }\end{array}$ & $\begin{array}{l}1.38 \\
(1.13-1.69) \\
1.19 \\
(1.12-1.26) \\
1.30 \\
(1.06-1.60) \\
- \\
-\end{array}$ & $\begin{array}{l}0.002 \\
<0.001 \\
0.013\end{array}$ & $\begin{array}{l}2.02 \\
(1.52-2.69) \\
1.10 \\
(1.04-1.17) \\
- \\
1.17 \\
(1.11-1.22) \\
-\end{array}$ & $\begin{array}{l}<0.001 \\
0.003\end{array}$ & $\begin{array}{l}1.70 \\
(1.28-2.25) \\
1.36 \\
(1.26-1.46) \\
- \\
- \\
1.82 \\
(1.35-2.46)\end{array}$ & $\begin{array}{l}<0.001 \\
<0.001\end{array}$ \\
\hline
\end{tabular}

\section{DISCUSSION}

Primary care patients who completed a pre-visit automated BI were more likely to: discuss drinking with their PCP at the subsequent visit, report raising the issue themselves, and receive a provider recommendation about their drinking. These group differences pertained for individuals with or without a diagnosis of AUD. Heavier drinkers were more likely than lighter drinkers to report discussing alcohol, initiating the discussion, and receiving a provider recommendation. Participants with a college degree were more likely to discuss alcohol with their PCP, older patients were more likely to self-initiate, and individuals with AUD were more likely to receive a PCP recommendation. The IVR-BI did not increase patient-provider discussion of other behavioral health topics. Importantly, the IVR-BI was well tolerated, as evidenced by the $97 \%$ completion rate. For about half of participants, IVRBI stimulated self-reflection, and for about one-third of participants, it was a useful alternative to a provider discussion.

These results suggest that: the overall rate of patient-provider discussions of alcohol use in primary care practice can be increased by providing automated pre-visit education and advice about alcohol; patient-provider communication is affected by relevant demographic and diagnostic factors unrelated to the IVR BI intervention; PCPs are more likely to make

Table 3. Patient Discussions with Provider During Office Visit Following IVR-BI Intervention

\begin{tabular}{lllll}
\hline \hline $\begin{array}{l}\text { Topic of Discussion } \\
\text { with Provider }\end{array}$ & $\boldsymbol{N}^{*}$ & Control & IVR-BI & $\boldsymbol{p}$ value \\
\hline alcohol use & 1567 & $44 \%$ & $52 \%$ & 0.003 \\
pain & 348 & $89 \%$ & $91 \%$ & 0.63 \\
tobacco use & 171 & $72 \%$ & $71 \%$ & 0.89 \\
physical activity & 911 & $81 \%$ & $82 \%$ & 0.56 \\
weight or diet & 683 & $72 \%$ & $77 \%$ & 0.11 \\
mood & 379 & $73 \%$ & $72 \%$ & 0.82 \\
\hline
\end{tabular}

* Analyses were limited to subjects who endorsed each item at the time of their screen recommendations for patients with greater severity, and the IVR-BI effect is specific to discussions of alcohol.

Consistent with earlier studies of PCP delivery of lifestyle advice, PCPs in this study were less likely to speak with patients about alcohol, compared with other conditions. ${ }^{21-25}$ Alcoholism is stigmatized, even among health care providers, ${ }^{26,27}$ who may expect patients to object when the topic is raised $^{1,28,29}$ and to under-report consumption and problems. ${ }^{26,30}$ Anecdotally, we did occasionally field questions from patients about whether their provider thought they were "an alcoholic." Thus, a common misconception may be that a doctor would only be concerned about a patient's drinking if there was a suspected AUD. Further education of the medical community and the general public is warranted regarding the effects on medical conditions and disease management, not just AUD, that can result from exceeding the NIAAA threshold for low-risk alcohol consumption. ${ }^{31-33}$

\section{Limitations}

Data on what transpired during the clinic visit was based solely on patient self-report, and thus was subject to recall and impression management biases. Existing evidence for the validity of proxy measures for clinician behavior is limited; however, a review study concluded that patient report is more accurate than provider report or records review. ${ }^{34}$ Another study reported rates of patient recall of PCP-delivered health behavior advice ranging from $76 \%$ (for smoking cessation) to $17 \%$ (for STD prevention), with substantial variability due to the reason for the visit and the amount of time spent discussing the targeted behavior. ${ }^{35}$ Accuracy of patient reports declines rapidly with increasing time from the event. ${ }^{36}$ The close proximity of our interview to the PCP appointment (median = 1 day) limits the influence of recall bias.

In this study, we were unable to measure or control for contamination effects. While we hypothesized that the IVRBI effect on communication would pertain only to the individual randomized patient and his or her PCP at that visit, it is 
conceivable that participants in the IVR-BI condition who initiated the topic of alcohol consumption affected their provider's proclivity to discuss alcohol with future patients, both experimental and control. The effect of any such contamination would be to increase the number of provider discussions in the control group, thus reducing the observed difference between groups. Indeed, $44 \%$ of control participants reported discussing alcohol with their providers, which is higher than other reports, ${ }^{37,38}$ and unexpectedly high considering PCPs had not been given any explicit instruction to intervene about alcohol during this study.

In this paper, our focus is on implementation rather than clinical outcomes. It remains to be seen whether either the IVR-BI and/or alcohol discussions as we have defined them are associated with improved clinical outcomes. Our sample consisted of predominately white, educated men and women living in a small metropolitan area. It is unknown how our findings would generalize to more diverse and urban settings. Unlike other technology platforms, IVR is highly accessible, inexpensive, and easy to use.

\section{Implications}

Our findings suggest that automated BIs are an important avenue to increase patient/provider discussion of alcoholrelated topics. Our data do not allow us to determine whether a full IVR-BI is required as a patient prompt, or whether IVR assessment + simple feedback would be sufficient. ${ }^{39,40}$ Regardless of its intensity, incorporating an alcohol-related IVR component into a standard appointment reminder call would be relatively straightforward. A strength of this study was that screening data were seamlessly merged with the EMR and available to the PCP at the time of the visit. A primary care system employing IVR and EMR, accessible at the point of care, could direct clinicians and patients to effective, evidence-based treatment options. ${ }^{41}$

\section{Acknowledgments:}

Contributors: We are grateful to the participating providers and staff in the Departments of Family Medicine and Primary Care Internal Medicine at the University of Vermont Medical Center for facilitating subject recruitment for this project.

Funders: This research was supported by grant R01AA018658 from the National Institute on Alcohol Abuse and Alcoholism (Gail L. Rose, PI).

Prior Presentations: Data from this trial were presented at the annual meeting of the International Network on Brief Interventions for Alcohol and Other Drugs in Atlanta, GA, in 2015.

Corresponding Author: Gail L. Rose, Ph.D.; Department of Psychiatry, The University of Vermont, Mail Stop \#457 OH3, 1 So. Prospect St., Burlington, VT 05401, USA (e-mail: gail.rose@uvm.edu).

\section{Compliance with Ethical Standards:}

Conflict of Interest: The authors declare that they do not have a conflict of interest.

\section{REFERENCES}

1. Beich A, Gannik D, Malterud K. Screening and brief intervention for excessive alcohol use: qualitative interview study of the experiences of general practitioners. BMJ. 2002;325(7369):870.

2. Fortney J, Mukherjee S, Curran G, Fortney S, Han X, Booth BM. Factors associated with perceived stigma for alcohol use and treatment among at-risk drinkers. J Behav Health Serv Res. 2004;31(4):418-429.

3. Johnson M, Jackson R, Guillaume L, Meier P, Goyder E. Barriers and facilitators to implementing screening and brief intervention for alcohol misuse: a systematic review of qualitative evidence. J Public Health (Oxf). 2011;33(3):412-421. doi:10.1093/pubmed/fdq095.

4. Spandorfer JM, Israel Y, Turner BJ. Primary care physicians' views on screening and management of alcohol abuse: inconsistencies with national guidelines. J Fam Pract. 1999;48(11):899-902.

5. National Center on Addiction and Substance Abuse (CASA). Missed Opportunity: CASA National Survey of Primary Care Physicians and Patients on Substance Abuse. New York: Columbia University; 2000.

6. Seale JP, Velasquez MM, Johnson JA, Shellenberger S, von Sternberg $\mathbf{K}$, Dodrill C, et al. Skills-based residency training in alcohol screening and brief intervention: results from the Georgia-Texas "Improving Brief Intervention" Project. Subst Abus. 2012;33(3):261-271. doi:10.1080/ 08897077.2011 .640187

7. Seale JP, Shellenberger S, Boltri JM, Okosun IS, Barton B. Effects of screening and brief intervention training on resident and faculty alcohol intervention behaviours: a pre- post-intervention assessment. BMC Fam Pract. 2005;6:46. doi:10.1186/1471-2296-6-46.

8. Rose GL, Guth SE, Badger GJ, Plante DA, Fazzino TL, Helzer JE. Brief intervention for heavy drinking in primary care: role of patient initiation. J Addict Med. 2015. doi:10.1097/ADM.0000000000000141.

9. Rose GL, MacLean CD, Skelly J, Badger GJ, Ferraro TA, Helzer JE. Interactive voice response technology can deliver alcohol screening and brief intervention in primary care. J Gen Intern Med. 2010;25(4):340-344. doi:10.1007/s11606-009-1233-0.

10. Rose GLF TA, Skelly JM, Badger GJ, MacLean CD, Fazzino TL, Helzer JE. Feasibility of automated pre-screening for lifestyle and behavioral health risk factors in primary care. BMC Fam Pract. In press.

11. Rose GL, Skelly JM, Badger GJ, Maclean CD, Malgeri MP, Helzer JE. Automated screening for at-risk drinking in a primary care office using interactive voice response. J Stud Alcohol Drugs. 2010;71(5):734-738.

12. Smith PC, Schmidt SM, Allensworth-Davies D, Saitz R. Primary care validation of a single-question alcohol screening test. J Gen Intern Med. 2009;24(7):783-788. doi:10.1007/s11606-009-0928-6.

13. NIAAA, NIoAAaA. Helping Patients Who Drink Too Much: A Clinician's Guide. Washington, D.C: National Institutes of Health; 2005.

14. Vinson DC, Kruse RL, Seale JP. Simplifying alcohol assessment: two questions to identify alcohol use disorders. Alcohol Clin Exp Res. 2007;31(8):1392-1398. doi:10.1111/j.1530-0277.2007.00440.x.

15. Lapham GT, Rubinsky AD, Shortreed SM, Hawkins EJ, Richards J, Williams EC, et al. Comparison of provider-documented and patientreported brief intervention for unhealthy alcohol use in VA outpatients. Drug Alcohol Depend. 2015;153:159-166. doi:10.1016/j.drugalcdep.2015.05.027.

16. Burman ML, Kivlahan D, Buchbinder M, Broglio K, Zhou XH, Merrill Jo, et al. Alcohol-related advice for Veterans Affairs primary care patients: Who gets it? Who gives it? J Stud Alcohol. 2004;65(5):621-630.

17. Bradley KA, Williams EC, Achtmeyer CE, Volpp B, Collins BJ, Kivlahan DR. Implementation of evidence-based alcohol screening in the Veterans Health Administration. Am J Manag Care. 2006;12(10):597-606.

18. Sobell LC, Sobell MB. Timeline follow-back: A technique for assessing selfreported alcohol consumption. In: Litten RZ, Allen JP, eds. Measuring Alcohol Consumption: Psychosocial and Biochemical Methods. Totowa: Humana Press; 1992:41-74.

19. Cottler LB, Robins LN, Helzer JE. The reliability of the CIDI-SAM: a comprehensive substance abuse interview. Br J Addict. 1989;84(7):801-814.

20. SAS Institute I. SAS. 9.4 ed. Cary: SAS Institute, Inc.; 2013.

21. Noordman J, Verhaak P, van Dulmen S. Discussing patient's lifestyle choices in the consulting room: analysis of GP-patient consultations between 1975 and 2008. BMC Fam Pract. 2010;11:87. doi:10.1186/ 1471-2296-11-87.

22. Duaso $\mathbf{M J}$, Cheung $\mathbf{P}$. Health promotion and lifestyle advice in a general practice: what do patients think? J Adv Nurs. 2002;39(5):472-479.

23. Johansson K, Bendtsen $\mathbf{P}$, Akerlind I. Advice to patients in Swedish primary care regarding alcohol and other lifestyle habits: how patients report the actions of GPs in relation to their own expectations and satisfaction with the consultation. Eur J Pub Health. 2005;15(6):615620. doi:10.1093/eurpub/cki046. 
24. Wallace PG, Brennan PJ, Haines AP. Are general practitioners doing enough to promote healthy lifestyle? Findings of the Medical Research Council's general practice research framework study on lifestyle and health. Br Med J (Clin Res Ed). 1987;294(6577):940-942.

25. McElwaine KM, Freund M, Campbell EM, Slattery C, Wye PM, Lecathelinais C, et al. Clinician assessment, advice and referral for multiple health risk behaviors: prevalence and predictors of delivery by primary health care nurses and allied health professionals. Patient Educ Couns. 2014;94(2):193-201. doi:10.1016/j.pec.2013.10.017.

26. Mules T, Taylor J, Price R, Walker L, Singh B, Newsam P, et al. Addressing patient alcohol use: a view from general practice. J Prim Health Care. 2012;4(3):217-222.

27. Ronzani TM, Higgins-Biddle J, Furtado EF. Stigmatization of alcohol and other drug users by primary care providers in Southeast Brazil. Soc Sci Med. 2009;69(7):1080-1084. doi:10.1016/j.socscimed.2009.07.026.

28. Friedmann PD, McCullough D, Chin MH, Saitz R. Screening and intervention for alcohol problems. A national survey of primary care physicians and psychiatrists. J Gen Intern Med. 2000;15(2):84-91.

29. McManus S, Hipkins J, Haddad P, Guthrie E, Creed F. Implementing an effective intervention for problem drinkers on medical wards. Gen Hosp Psychiatry. 2003;25(5):332-337.

30. Lock CA, Kaner E, Lamont S, Bond S. A qualitative study of nurses attitudes and practices regarding brief alcohol intervention in primary health care. J Adv Nurs. 2002;39(4):333-342.

31. Bradley KA, Rubinsky AD, Williams EC, Lapham GT, Achtmeyer CE, Kivlahan DR. Using screening scores to provide feedback to patients on their alcohol-related risks: the association between AUDIT-C scores and average consumption and health outcomes. International Network on Brief Interventions for Alcohol and Other Drugs (INEBRIA); 19-20 September 2013; Rome, Italy: Addiction Science \& Clinical Practice; 2013. p. A13.

32. Bradley KA, Badrinath S, Bush K, Boyd-Wickizer J, Anawalt B. Medical risks for women who drink alcohol. J Gen Intern Med. 1998;13(9):627-639.
33. Room R, Babor T, Rehm J. Alcohol and public health. Lancet. 2005;365(9458):519-530. doi:10.1016/S0140-6736(05) 17870-2.

34. Hrisos S, Eccles MP, Francis JJ, Dickinson HO, Kaner EF, Beyer F, et al. Are there valid proxy measures of clinical behaviour? A systematic review. Implement Sci. 2009;4:37. doi:10.1186/1748-5908-4-37.

35. Flocke SA, Stange KC. Direct observation and patient recall of health behavior advice. Prev Med. 2004;38(3):343-349. doi:10.1016/j.ypmed. 2003.11.004.

36. Ward J, Sanson-Fisher R. Accuracy of patient recall of opportunistic smoking cessation advice in general practice. Tob Control. 1996;5(2):110113.

37. Funk M, Wutzke S, Kaner E, Anderson P, Pas L, McCormick R, et al. A multicountry controlled trial of strategies to promote dissemination and implementation of brief alcohol intervention in primary health care: findings of a World Health Organization collaborative study. J Stud Alcohol. 2005;66(3):379-388.

38. Silagy C, Muir J, Coulter A, Thorogood M, Yudkin P, Roe L. Lifestyle advice in general practice: rates recalled by patients. BMJ. 1992;305(6858):871-874.

39. Kaner E, Bland M, Cassidy P, Coulton S, Dale V, Deluca P, et al. Effectiveness of screening and brief alcohol intervention in primary care (SIPS trial): pragmatic cluster randomised controlled trial. BMJ. 2013;346:e8501. doi:10.1136/bmj.e8501.

40. Kuerbis AN, Yuan SE, Borok J, LeFevre PM, Kim GS, Lum D, et al Testing the initial efficacy of a mailed screening and brief feedback intervention to reduce at-risk drinking in middle-aged and older adults: the comorbidity alcohol risk evaluation study. J Am Geriatr Soc. 2015;63(2):321-326. doi:10.1111/jgs. 13237.

41. Stacey D, Legare F, Col NF, Bennett CL, Barry MJ, Eden KB, et al. Decision aids for people facing health treatment or screening decisions. Cochrane Database Syst Rev. 2014;1:CD001431. doi:10.1002/14651858. CD001431.pub4. 